\title{
Can a non chiral object be made of two identical chiral moieties?
}

J. Chem. Educ. 2008, 85, 433-435.

Jean François LeMARECHAL

Université de Lyon, École Normale Supérieure de Lyon, 46 allée d'Italie, 69364, LYON, FRANCE

\begin{abstract}
Several pedagogical objects can be used to discuss chirality. Here, we use the cut of an apple to show that the association of identical chiral moieties can form a non chiral object. Octahedral chirality is used to find situations equivalent to the cut of the apple.
\end{abstract}

Key words: Second-Year Undergraduate, Inorganic Chemistry, Analogies, Coordination Compounds, Chirality, Octahedral chirality, Enantiomorph, Symmetry, King's cut

\section{Chirality}

It is commonplace when teaching chirality to use material objects to illustrate the ideas involved in this domain of chemistry. Chirality is the property of molecules or objects which are non superimposable on their mirror-image. While pairs of chiral molecules are called enantiomers, pairs of chiral material objects, such as crystals, are called enantiomorphs (1). As far as molecules are concerned, chirality leads to the property of being optically active, which consists in being able to rotate the plane of polarized light. Several examples of material objects used to help teach the concept of superimposability have been presented in this Journal. This concept, which is pivotal for learning chirality, has been introduced by asking students to consider the properties of two identical books that can lead to the conclusion that the books are, indeed, the same (3). Other examples of pedagogical tools are plain identical dishes that have also been used to introduce the notion of superimposability. Their stacking makes superimposability apparent and they can be differentiated by marking them (4). The same source also uses nautilus shells split in half to illustrate enantiomorphs. In this article, we will use the example of cutting apples as a metaphor for the association of chiral centers.

The chirality of an object can be identified by comparing it with its mirror-image (5), but a more straightforward way is to use symmetry considerations. Molecules that have planes, centers, or alternating axes cannot be chiral (2), and most of the time, only the symmetry plane operation is considered. It can also be helpful for students to learn how to recognize the possible chiral centers of molecules.

At school, chirality is often introduced with asymmetrical carbon atoms, which are basic and frequently occurring chiral centers, but many other situations may arise. The phosphorous atom in phosphine may be a chiral center and molecules may present a chiral axis, such as in allenes, biphenyles or spiranes. Molecules with a helical structure also exist, such as [n] helicene $(n \geq 6)$ or molecules of higher complexity such as starch (6), DNA or proteins. In inorganic complexes, Werner demonstrated that octahedral complexes could be chiral (7-9) by resolving $\mathrm{Co}\left(\mathrm{NH}_{3}\right)(\mathrm{Cl})\left(\mathrm{H}_{2} \mathrm{NCH}_{2} \mathrm{CH}_{2} \mathrm{NH}_{2}\right)_{2}$ then, later, $\mathrm{Co}_{4}(\mathrm{OH})_{6}\left(\mathrm{NH}_{3}\right)_{12}$. He even proved with the latter that enantiomery could occur in species that contain no carbon atoms.

The association of two chiral centers within one molecule can lead to interesting cases. When two fragments of a molecule are both chiral and mirror images, for example meso-tartaric 
acid, the whole molecule is not chiral. The first part of this paper questions the converse of the previous statement, i.e., it asks, if a non chiral molecule is made of two chiral centers, does it follow that these centers must be mirror images of each other? In other words, we will look for a counterexample that shows how a non chiral object such as an apple can be split into two identical chiral objects. Then, in the second part of the paper, we will compare the parts of the apple with octahedral complexes.

\section{The "King's cut"}

Among the ways in which people cut their apples, one deserves special attention, as it leads to two elegant portions that fit together as in a three dimensional puzzle. This way of cutting apples is named the King's cut due to the legend that it was presented to a King in a remote country. To carry out a King's cut, start by cutting a vertical quarter down from the stalk of the apple, marked $\mathrm{N}$ in figure 1.a (along the line $\mathrm{NA}$ ), then cut a horizontal half (line $\mathrm{ABC}$ ), then a vertical quarter (line CS) down to the bottom of the fruit, then a last half (line SDN) back to the stalk. This cut will be considered either as the six arcs NA, AB, BC, CS, SD and $\mathrm{DN}$, or as the NABCSDN line in the following. None of these cuts should cross each other. If the blade of the knife cuts the apple as deeply as the middle axis of the fruit, but not more, the two pieces can be separated. Beginners might break the apple or get non perpendicular pieces, but practice soon makes perfect.

(a)

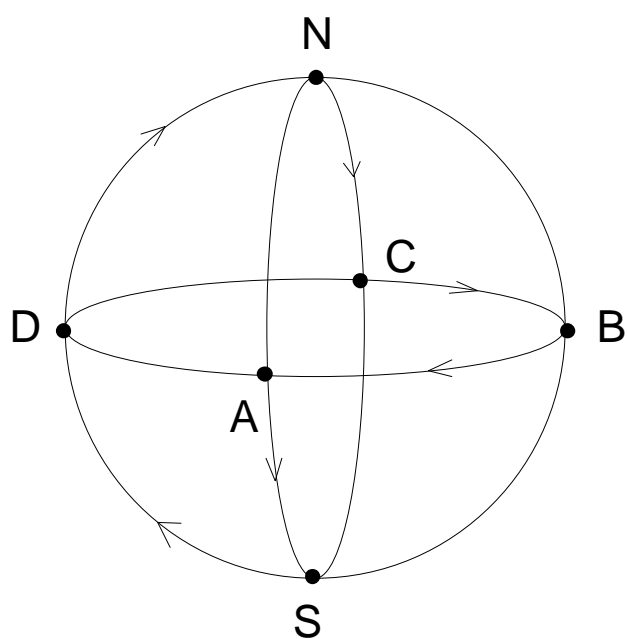

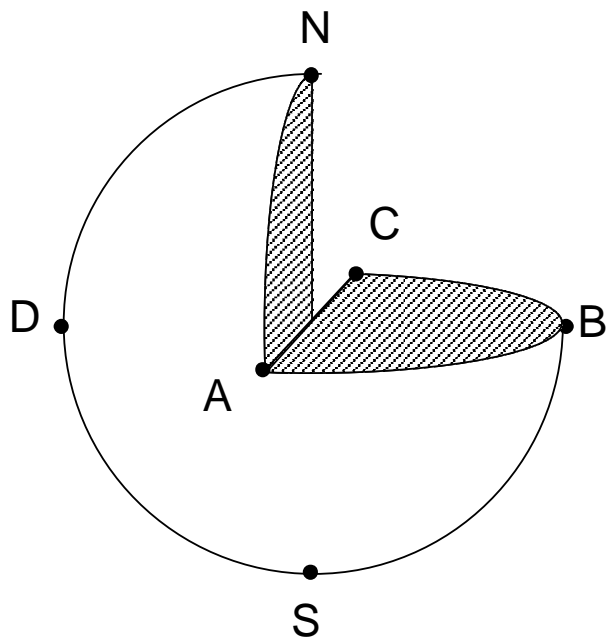

S

Fig. 1 - From the sphere to the cut pieces of the apple.

Observing one of the pieces should show that it is chiral, even assuming the apple to be a perfect sphere. It has no symmetry plane, no improper axis, and there is not even any symmetry operation other than a $\mathrm{C} 2$ axis. The $\mathrm{C} 2$ axis passes from the middle of the line NC through the centre, and out again at the middle of line SA. Therefore it belongs to the chiral point group $\mathrm{C}_{2}$. Observing both pieces proves that they are identical, provided the apple is assumed to be spherical, and the King's cut was properly carried out. Both are made of two quarters of an apple perpendicularly assembled in the same way (Fig. 1b). The enantiomorphous pieces could have been produced by performing the other King's cut: NADCSBN (Fig. 2). 


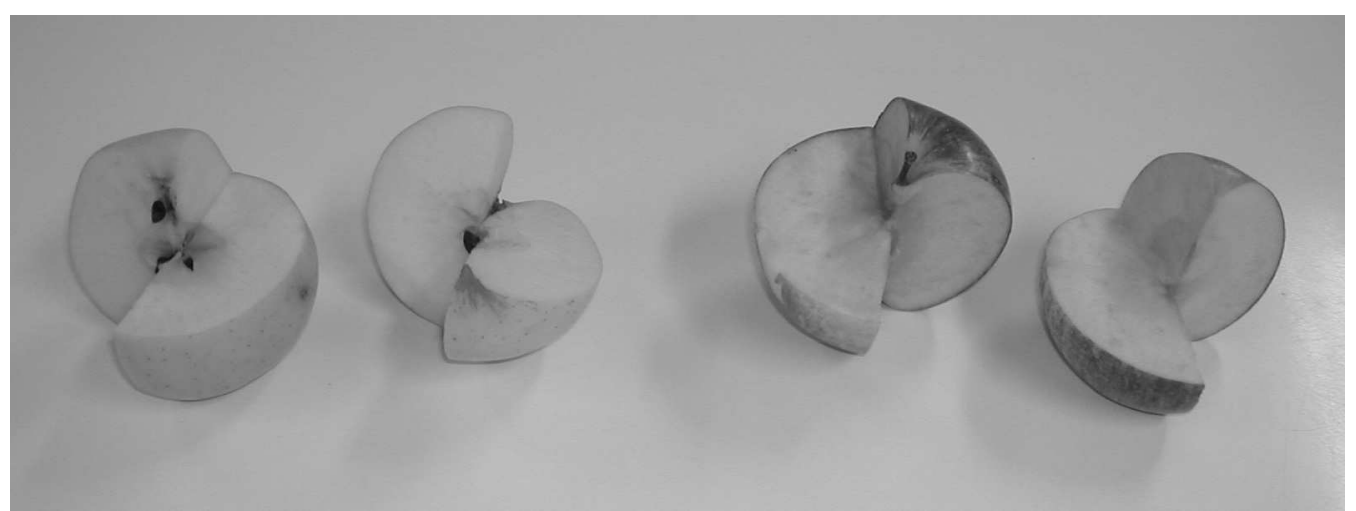

Fig. 2 - The result of the two possible King's cuts. The two pieces of the yellow apple are chiral and identical, and mirror images of the pieces of the red apple.

The fascinating thing about the King's cut is that it is the counterexample we mentioned earlier, as it arises from the fact that an achiral object (the apple) can be partitioned into two identical chiral pieces. A usual and easy partition of an achiral object (say a human body) leads to a left and a right part. Both chiral shares are enantiomorphs. In the case of the King's $c u t$, the pieces are the same. In the world of molecules, a meso stereoisomer such as the mesotartaric acid is made of an $\mathrm{R}$ chiral center and an $\mathrm{S}$ one; not two $\mathrm{S}$ ones. The King's cut is therefore highly intriguing.

\section{Molecular chirality and King's cut}

Octahedral complexes have a few features in common with a King's cut. Complexes made of three chelating dihapto ligands, such as ethylendiamine (en), are often found in undergraduate textbooks. However, there are also other kinds of chiral octahedral complexes, some of which are discussed below. Their relation to the King's cut is examined.

The simplest ones are $\mathrm{M}(\mathrm{en})_{3}$ or $\mathrm{M}\left(\mathrm{CH}_{3} \mathrm{OCH}_{2} \mathrm{CH}_{2} \mathrm{OCH}_{3}\right)_{3}$, as in Fig. 3. Their chirality always takes some time to be evident to students, and it is often necessary to represent them along the $\mathrm{C}_{3}$ axis of the complexes. Such complexes belong to point group $\mathrm{D}_{3}$, as the only symmetry operations are three $\mathrm{C}_{2}$ axes perpendicular to the main $\mathrm{C}_{3}$ axis. The nomenclature of these complexes uses the $\Delta / \Lambda$ notations (10). In the same family, complexes such as $\mathrm{M}\left(\mathrm{CH}_{3} \mathrm{OCH}_{2} \mathrm{CH}_{2} \mathrm{~N}\left(\mathrm{CH}_{3}\right)_{2}\right)_{3}$ are chiral but with an even lower symmetry, as there is only a $\mathrm{C}_{3}$ axis. A complex of this sort belongs to group point $\mathrm{C}_{3}$. The similarity of chiral complexes of this kind with the apple pieces can be seen from the number of common spherical arcs involved: NA, BC and SD (Fig. 1). These arcs match three of the six of the King's cut (NA, $\mathrm{AB}, \mathrm{BC}, \mathrm{CS}, \mathrm{SD}$, and $\mathrm{DN}$ ). We looked for other ligands that could fit more. 

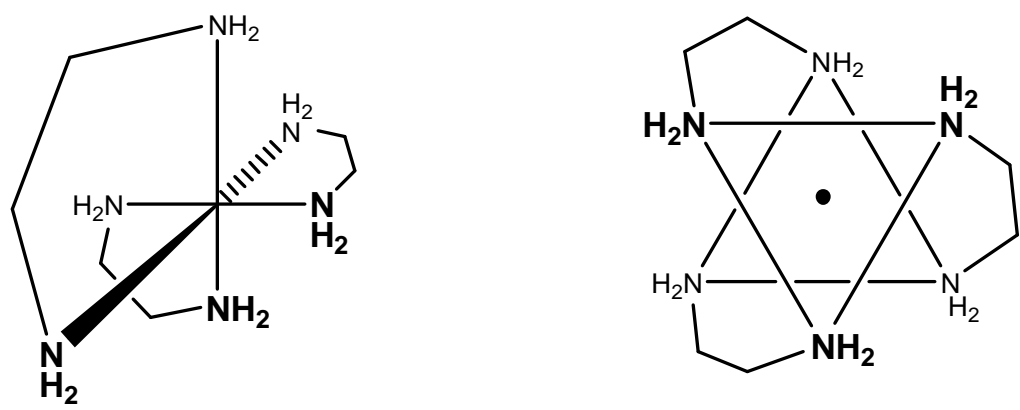

Fig. 3 - The octahedral structure of $\mathrm{M}(\mathrm{en})_{3}$ and its view from the $\mathrm{C}_{3}$ axis. Two bonds are enlarged for the sake of clarity, but the octahedron is regular. The $\mathrm{NH}_{2}$ fragments presented in bold face correspond in the two drawings.

The Ca(edta $)^{2+}$ complex is more famous for its analytic use than for its chiral property. It differs from the $M(e n)_{3}$ chirality in the topology of the ligand since only one ligand wraps up the metal cation. The structure of such a complex was obtained from $\mathrm{Ca}$ [Ca(edta)]. $7 \mathrm{H}_{2} \mathrm{O}$ (11) and compared with others (12). The angles of the bonding around the Ca cation are far from the $90^{\circ}$ in the crystal, but in the propylendiaminetetraacetate (pdta) ion, the structure is almost octahedral (Fig. 4). With the notation of the King's cut, the complex can be represented by the $\mathrm{NA}, \mathrm{DA}, \mathrm{AB}, \mathrm{BC}$, and $\mathrm{BD}$ arcs of the sphere. Although there are more arcs than with the $\mathrm{M}(\mathrm{en})_{3}$ complex, the topology of the complex is still different from the King's cut.

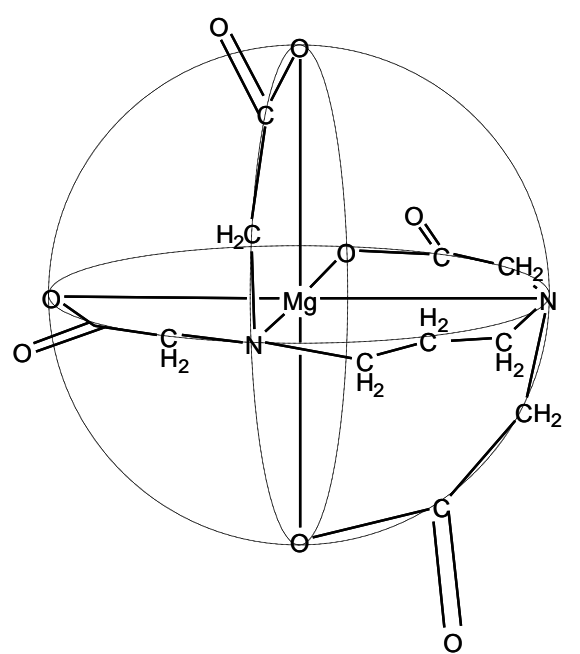

Fig. 4 -Drawing of $\mathrm{Mg}$ (pdta) from an ORTEP representation (12) showing a reasonable octahedral chiral complex with a single ligand wrapping up the cation.

The last complex to be examined here might be expected to be the closest to the King's cut, as the topology of the ligand is a linear chain just like the trace of the blade of the knife on the skin of the apple. The ligand is called pentaglyme, $\mathrm{CH}_{3}\left(\mathrm{OCH}_{2} \mathrm{CH}_{2}\right)_{5} \mathrm{OCH}_{3}$, from which calcium and strontium complexes were prepared and X-ray characterized (13). Five possible geometries can a priori be considered for a hexadentate linear ligand wrapping up a cation at the $\mathrm{N}, \mathrm{A}, \mathrm{B}, \mathrm{C}, \mathrm{D}$, and $\mathrm{S}$ octahedral positions on the sphere (using the notation of Fig. 1). These geometries can be encoded as NABCDS; NABCSD; NABSCD; NABSDC; NASBCD (Fig.5). The enantiomeric structures can also be considered. Although these five structures are relatively easy to identify, proving that there are only five of them is difficult. Unfortunately, the thiocyanate counter anions also coordinate the calcium cation as shown by the crystal structure. The $\mathrm{Ca}$ (pentaglyme)(NCS) ${ }_{2}$ complex gets both thiocyanato ligands in trans positions (such as $\mathrm{N}$ and $\mathrm{S}$ in Fig. 1). The complex structure is close to a distorted NABCDS 
King's cut, with the first and sixth oxygen atoms of the chain close (above an below) to the $\mathrm{ABCD}$ plan, and the 2-5 oxygen in this plan.

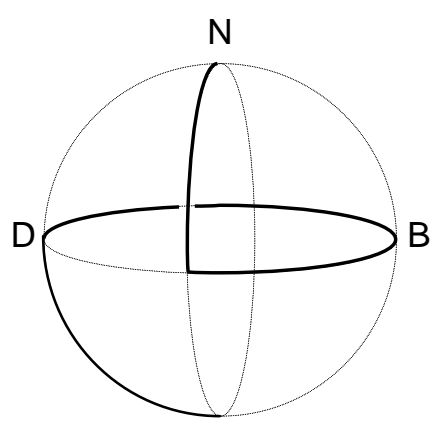

S

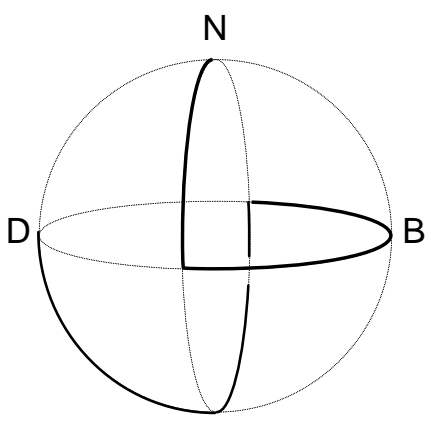

$\mathrm{S}$

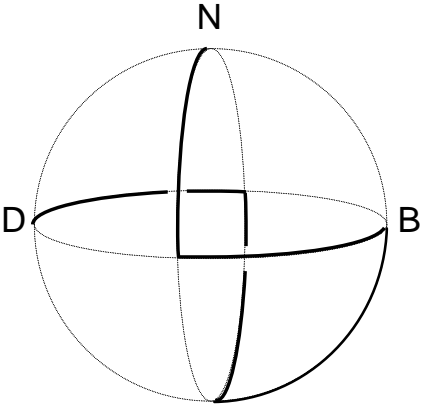

$\mathrm{S}$

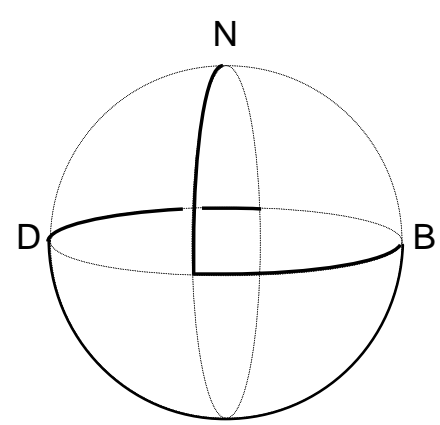

S

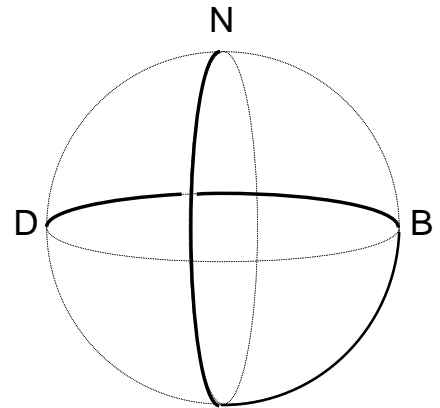

$\mathrm{S}$

Fig. 5 - Possible structures for an octahedral complex with a linear hexadentate ligand. The points A (front) and C (back) are not indicated for the sake of clarity.

Our initial intention was to question the converse proposition according to which the association of two enantiomeric moieties should lead to an achiral molecule, as in mesotartaric acid. We were inspired by the example of the King's cut to find molecular topologies similar to it. Among the typology of chiral centers (tetrahedral, chiral axis, etc.), we focused on octahedral chirality, which looks close to the apple cut. We examined three topologies of ligands. Each of them led to a chiral complex that was too dissimilar from the King's cut. We are therefore led to believe that there are no molecular examples analogous to the King's cut, which will probably remain a unique counterexample.

Cutting an apple a day may keep symmetry away.

I thank Anne Paupe and Robin Millar for improving the manuscript of this article and for the fruitful discussion.

\section{Literature cited}

1. Eliel, E. L.; Wilen, S. H. Stereochemistry of Organic Compounds. John, Wiley \& Sons, INC. New-York 1994, p. 1198.

\section{2. ibid. p. 58 .}

3. Lujàn-Upton, H. J. Chem. Educ. 2001, 78, 475-477.

4. Hart, H. J. Chem. Educ. 2001, 78, 1632-1634.

5. Collins, M. J. J. Chem. Educ. 2001, 78, 1484-1485. 
6. Hancock, R. D. J. Chem. Educ. 2000, 77, 988-992.

7. Huheey, J.E. Inorganic Chemistry. $3^{\text {rd }}$ Ed. Harper \& Row, New-York, 1983, p. 493.

8. Werner, A. Ber. 1911, 44, 1887-1898.

9. Werner, A. Ber. 1912, 45, 121-130.

10. Herrero, S.; Uson, M.A. J. Chem. Educ. 1995, 72, 1065-1066.

11. Barnett, B.L.; Uchtman, V.A. Inorg.Chem. 1979, 18 (10), 2674.

12. Radanović, D. D.; Rychlewska U.; Djuran, M. I.; Warżajtis, B.; Drašković, N. S.; Gurešić, D. M. Polyhedron, 2004, 23, 2183-2192

13. Wei, Y. Y.; Tinant, B.; Declercq, J.-P.; Van Meerssche, M.; Dale, J. Acta Cryst., 1987, C43, 1270-1274. 